\title{
Valutazione preliminare della presenza di 3-cheto-4-colestene in Parmigiano Reggiano D.O.P.
}

\section{Preliminary evaluation of 3-keto-4-cholestene in Parmigiano Reggiano P.D. O. cheese}

${ }^{1}$ Università degli Studi di Parma, Dipartimento di Scienze degli Alimenti e del Farmaco, Parco Area delle Scienze 27A, 43124, Parma, Italiaù

${ }^{2}$ Consorzio del formaggio Parmigiano Reggiano, Via J.F. Kennedy 18, 42124 Reggio Emilia, Italia

*Corresponding author:

Valentina Pizzamiglio

Consorzio del formaggio Parmigiano Reggiano, Via J.F. Kennedy 18, 42124 Reggio Emilia pizzamiglio@parmigianoreggiano.it +390522307741
Ricevuto il 3 settembre 2019

Accettato il 2 ottobre 2019

DOI: $10.36138 /$ STLC.01.2019.02

\section{Riassunto}

La percentuale massima di crosta in Parmigiano Reggiano grattugiato è definita dal Disciplinare di produzione al 18\%. Lo scopo del presente studio è valutare la presenza di 3-cheto-4-colestene in campioni di pasta di Parmigiano Reggiano e di crosta di differenti stagionature (12 e 24 mesi) e sottoposte a different metodi di pulizia (lavaggio ad acqua e raschiatura a secco).

Lo studio ha riguardato 31 campioni di crosta e 10 campioni di pasta interna mediante GC-MS, rilevando il rapporto tra l'area del 3-cheto4-colestene e del colesterolo. Dai dati preliminari ottenuti è possibile ipotizzare che l'assenza di questa molecola in campioni provenient dalla parte interna della forma di Parmigiano Reggiano e la presenza in campioni di crosta, possa essere utilizzata per discriminare queste due tipologie di campioni. In merito alle altre variabili (stagionatura e metodo di pulizia), il ridotto numero di campioni e l'elevato livello di variabilità tra i campioni dello stesso gruppo, non permette di definire una correlazione significativa e rende necessari ulteriori studi.

\section{Parole chiave:}

- Parmigiano Reggiano

FormaggioF

- Formaggio grattugiato

- Crosta

3-cheto-4-colestene

\section{Abstract}

The maximum percentage of cheese rind in Parmigiano Reggiano P.D.O. grated cheese is fixed at $18 \%$ by the product Specification. Purpose of the present study is to evaluate the presence of 3-keto4-cholestene in grated Parmigiano Reggiano cheese and rind in different ages (12 and 24 months) and from different cleaning treatments of the rind (washing by water and dry scraping of the rind). The study analysed 31 samples of cheese rind and 10 samples of cheese by GC-MS, detecting the ratio between the area of 3-keto-4-cholestene and cholesterol. Preliminary data suggested that the absence of this molecule in the samples from the inner part of the wheel and its presence in the samples from the rind, could be used to discriminate these two kinds of samples. Regarding other variables (ages and cleaning treatment), due to low number of samples and high degree of variability among samples from the same group, it is not possible to define a significative correlation and further studies are needed.

\section{Keywords:}

- Parmesan

Cheese

Grated cheese

Rind

3-keto-4-cholestene 
Tabella I: Rapporto area 3-cheto-4-colestene /area colesterolo (m/z 124) per i campioni di crosta analizzati di diverse stagionature (12-24 mesi) e sottoposti a diversi trattamenti di pulizia (a secco, ad acqua)

Table l: Area ratio 3-keto-4-cholestene/ cholesterol ( $\mathrm{m} / \mathrm{z}$ 124), rind samples from different age (12-24 months) and different cleaning (dry cleaning, water cleaning)

\begin{tabular}{|c|c|c|c|}
\hline Campione & $\begin{array}{l}\text { Stagionatura } \\
\text { (mesi) }\end{array}$ & Pulizia forme & $\begin{array}{c}\text { area 3-cheto- } \\
\text { 4-colestene } \\
\text { / area } \\
\text { colesterolo } \\
\text { (m/z 124) }\end{array}$ \\
\hline 1 & 12 & Raschiatura a secco & 1,72 \\
\hline 2 & 12 & Raschiatura a secco & 0,86 \\
\hline 3 & 12 & Raschiatura a secco & 1,82 \\
\hline 4 & 12 & Raschiatura a secco & 1,18 \\
\hline 5 & 12 & Raschiatura a secco & 0,44 \\
\hline 6 & 12 & Raschiatura a secco & 3,58 \\
\hline 7 & 12 & Raschiatura a secco & 1,49 \\
\hline 8 & 12 & Raschiatura a secco & 1,73 \\
\hline 9 & 12 & Raschiatura a secco & 1,44 \\
\hline 10 & 12 & Raschiatura a secco & 4,60 \\
\hline 11 & 12 & Raschiatura a secco & 3,66 \\
\hline 12 & 12 & Raschiatura a secco & 8,96 \\
\hline 13 & 12 & Raschiatura a secco & 7,57 \\
\hline 14 & 12 & Raschiatura a secco & 0,83 \\
\hline \multirow[t]{3}{*}{15} & 12 & Raschiatura a secco & 0,54 \\
\hline & & Media & 2,69 \\
\hline & & Deviazione standard & 2,58 \\
\hline
\end{tabular}

\begin{tabular}{cccc} 
Campione & $\begin{array}{c}\text { Stagionatura } \\
\text { (mesi) }\end{array}$ & Pulizia forme & $\begin{array}{c}\text { area 3-cheto- } \\
\text { 4-colestene } \\
\text { / area } \\
\text { colesterolo } \\
\text { (m/z 124) }\end{array}$ \\
\hline 16 & 24 & Raschiatura a secco & 0,68 \\
\hline 17 & 24 & Raschiatura a secco & 3,13 \\
\hline 18 & 24 & Raschiatura a secco & 0,97 \\
\hline 19 & 24 & Raschiatura a secco & 3,91 \\
\hline 20 & 24 & Raschiatura a secco & 7,04 \\
\hline 21 & 24 & Raschiatura a secco & 4,40 \\
\hline & & Media & 3,36 \\
& & Deviazione standard & 2,36
\end{tabular}

\begin{tabular}{cccc}
22 & 12 & Lavaggio ad acqua & 0,77 \\
\hline 23 & 12 & Lavaggio ad acqua & 0,91 \\
\hline 24 & 12 & Lavaggio ad acqua & 1,63 \\
\hline 25 & 12 & Lavaggio ad acqua & 9,06 \\
\hline & & Media & 3,09 \\
& & Deviazione standard & 4,00
\end{tabular}

\begin{tabular}{cccc}
26 & 24 & Lavaggio ad acqua & 5,08 \\
\hline 27 & 24 & Lavaggio ad acqua & 1,78 \\
\hline 28 & 24 & Lavaggio ad acqua & 7,13 \\
\hline 29 & 24 & Lavaggio ad acqua & 3,64 \\
\hline 30 & 24 & Lavaggio ad acqua & 10,65 \\
\hline 31 & 24 & Lavaggio ad acqua & 36,60 \\
\hline & & Media & 10,81 \\
& & Deviazione standard & 13,00
\end{tabular}

Reggiano" anche per la tipologia di formaggio grattugiato ottenuta esclusivamente da formaggio che detiene già tutti i requisiti per la D.O.P. e a condizione che le operazioni di grattugiatura siano effettuate nell'ambito territoriale di produzione del Parmigiano Reggiano e che il confezionamento avvenga senza alcun trattamento e senza aggiunta di sostanze atte a modificare la conservabilità e le caratteristiche organolettiche originarie. La denominazione è riservata al formaggio grattugiato avente anche i seguenti requisiti: umidità compresa tra 25-35\%; aspetto non pulverulento ed omogeneo, particelle con diametro inferiore a $0,5 \mathrm{~mm}$ non superiori al $25 \%$; quantità di crosta non superiore al 18\%. II Consorzio, nell'ambito delle sue attività di ricerca al servizio delle attività di tutela e vigilanza, è coinvolto nello studio e nel miglioramento continuo di metodi-

che analitiche per la determinazione dell'autenticità del prodotto e per la quantificazione dei parametri descritti nel Disciplinare di produzione.

In riferimento a quest'ultimo parametro, risultati preliminari condotti su alcune produzioni alpine italiane locali di formaggi a pasta dura e semidura di Dobbiaco (Tre Cime) (BZ), Bagolino (Bagoss) (BS) e Livinallongo (Fodom) (BL) hanno mostrato che il contenuto di 3-cheto-4-colestene sulla crosta del formaggio aumenta regolarmente con l'invecchiamento e diminuisce spostando il campiona- 


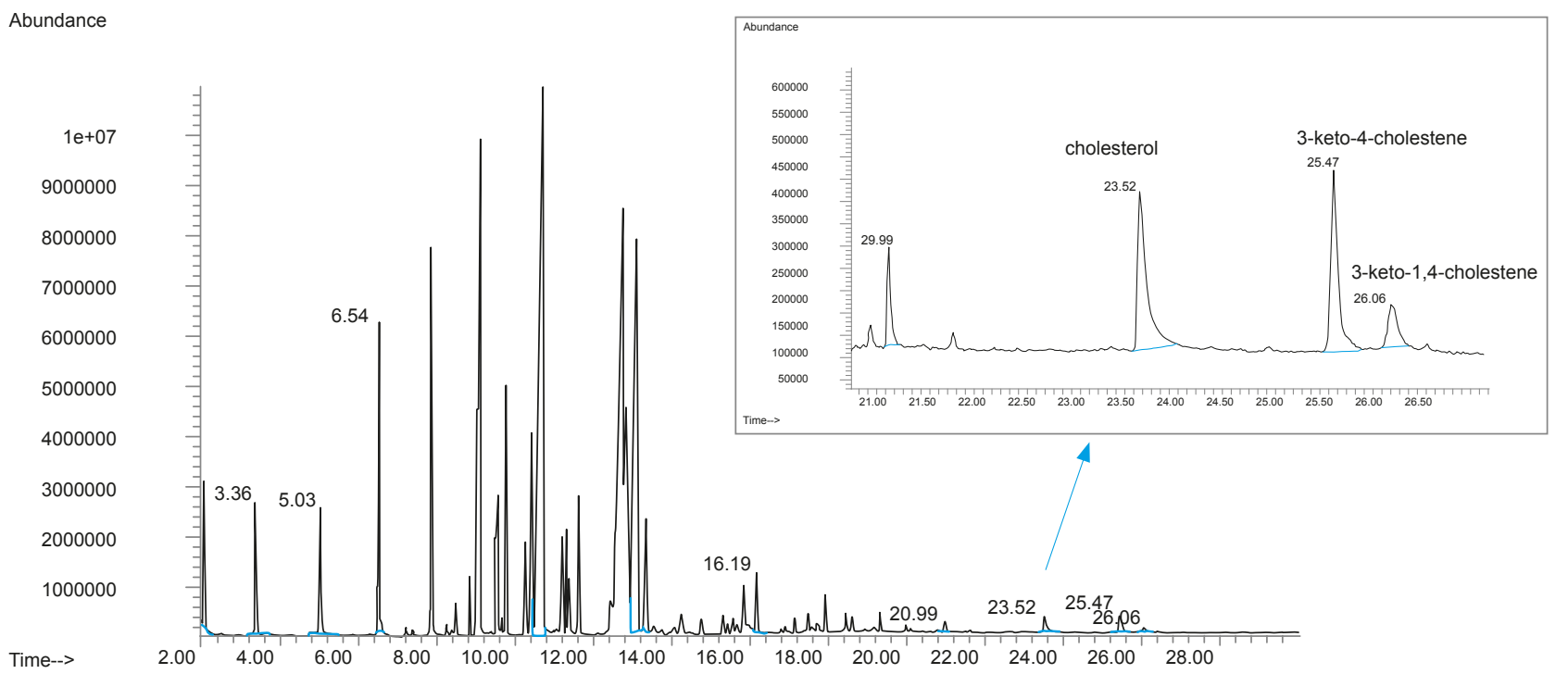

Figura 1: (a) analisi GC-MS del profilo lipidico di una crosta di formaggio (1-2 mm) in modalità SCAN (b) dettaglio della zona di eluizione (frammento estratto $\mathrm{m} / \mathrm{z} 124)$ di colesterolo (23,62 minuti), 3-keto-colestene $(25,47$ minuti) e 3-keto-1,4colestandiene (26,05 minuti)

Figure 1: (a) GC-MS analysis of lipidic profile of Parmigiano Reggiano cheese rind (1-2 mm) in SCAN mode (b) detail of elution area (extracted fragment m/z 124), cholesterol (23,62 minute); 3-keto-4-cholestene (25,47 minute) and 3-keto-1,4cholestandiene (26,05 minute)

mento dalla parte esterna alla parte interna della forma, suggerendo la possibilità di considerare questa molecola come un possibile indicatore molecolare dell'età del formaggio e/o della percentuale di crosta presente nel formaggio grattugiato (2). La presenza di questa sostanza, che fa parte della categoria dei prodotti di ossidazione del colesterolo, può essere correlata all'enzima colesterolo ossidasi di muffe e batteri che crescono sul formaggio, producendo 3-cheto-4-colestene e altri ossidi che si accumulano gradualmente sulla crosta del formaggio $(3,4)$.

Nell'ottica di trovare un marker della presenza di crosta nei grattugiati, risulta interessante approfondire il ruolo di questa molecola come indicatore sulla percentuale di crosta contenuta nel Parmigiano Reggiano grattugiato.

Il presente studio si pone quindi l'obiettivo di valutare in via preliminare, il contenuto di 3-cheto-4-colestene in campioni di Parmigiano Reggiano di diverse stagionature e sottoposto a diversi metodi di pulizia, al fine di valutare l'eventuale utilizzo di questa molecola come indicatore della percentuale di crosta presente nel formaggio Parmigiano Reggiano grattugiato.

\section{MATERIALI E METODI}

Nel presente studio sono stati analizzati 31 campioni di croste di Parmigiano Reggiano suddivisi tra stagionatura 12 mesi (19 campioni) e 24 mesi (12 campioni). I campioni della stagionatura 12 mesi provenivano da forme pulite mediante lavaggio ad acqua (4 campioni) e da forme pulite mediante raschiatura a secco (15 campioni). I campioni della stagionatura 24 mesi provenivano da forme pulite mediante lavaggio ad acqua ( 6 campioni) e da forme pulite mediante raschiatura a secco (6 campioni). Sono stati inoltre analizzati 10 campioni di formaggio dopo aver rimosso i primi $2 \mathrm{~cm}$ di crosta.

Un'aliquota di 1,0 g di crosta di formaggio/pasta interna è stata accuratamente macinata e mescolata con miscela esano/acetone $\left(20 \mathrm{ml}, 4 / 1 \mathrm{v} / \mathrm{v}\right.$ ) riscaldando a circa $50^{\circ} \mathrm{C}$ per estrarre il grasso. L'estrazione è stata eseguita due volte. L'estratto è stato portato a secchezza in un evaporatore rotante a $50{ }^{\circ} \mathrm{C} .200 \mathrm{mg}$ del residuo grasso sono stati sciolti in esano $(2 \mathrm{ml})$ e trattati con $0,5 \mathrm{ml}$ di soluzione di idrossido di potassio (10\% in metanolo). Dopo aver miscelato, è stata raccolta la frazione esano superiore $(0,5 \mathrm{ml})$ e aggiunta a $0,2 \mathrm{ml}$ di HMDS e $0,1 \mathrm{ml}$ di TMCS. Dopo il riscaldamento $(70$ 
${ }^{\circ} \mathrm{C}$ in forno per 30 minuti) e la separazione delle fasi, è stata iniettata la fase organica superiore $(1 \mu \mathrm{l}$, modalità split) su un gascromatografo Agilent Technologies 6890N (Agilent Technologies, Palo Alto, CA, USA) accoppiato a uno spettrometro di massa Agilent Technologies 5973 (Agilent Technologies, Palo Alto, CA, USA). È stata utilizzata una colonna capillare a bassa polarità (SLB-5ms, Supelco, Bellafonte, USA). II cromatogramma è stato registrato in modalità SIM (m/z 74, 87, 124, 155, 174, 278, 368, 382, 384, 386, $412,430 \mathrm{~m} / \mathrm{z}$ ) con una temperatura programmata da $70^{\circ} \mathrm{C}$ a $290^{\circ} \mathrm{C}$ $\left(70^{\circ} \mathrm{C}\right.$ per 1 minuto, da $70^{\circ} \mathrm{C}$ a $220^{\circ} \mathrm{C}$ a $20^{\circ} \mathrm{C}$ e isoterma per $6 \mathrm{mi}-$ nuti, da $220^{\circ} \mathrm{C}$ a $290^{\circ} \mathrm{C}$ a $20^{\circ} \mathrm{C}$ e mantenuto a $290^{\circ} \mathrm{C}$ per 25 minuti). Le identità dei picchi di colesterolo, 3-cheto-4-colestene e 3-cheto-1,4-colestandiene sono state confermate dal confronto con standard puri. I risultati vengono forniti come rapporto tra le aree di 3-cheto-4-colestene/colesterolo dopo estrazione del frammento caratteristico 124 m/z (Fig. 1).

\section{RISULTATI}

In Tabella I sono riportati i dati dei singoli campioni di croste analizzati suddivisi per stagionatura (12-24 mesi) e tipologia di lavaggio (raschiatura a secco e lavaggio ad acqua) e la media e deviazione standard di ciascun gruppo di campioni.

I 10 campioni di formaggio analizzati dopo rimozione della crosta, hanno evidenziato assenza di 3-cheto-4-colestene in 9 campioni. Di questi, un solo campione di pasta ha mostrato presenza di 3-cheto-4-colestene, di conseguenza, un rapporto 3-cheto-4-colestene/colesterolo (124 m/z) pari a 0,04 (Tab. II).

In tabella III sono riportate le medie e relative deviazioni standard dei campioni analizzati suddivisi per stagionatura e metodo di pulizia della crosta.

Numericamente i campioni provenienti da croste di 24 mesi presentano un contenuto medio di 3-cheto-4-colestene più elevato rispetto ai campioni provenienti da forme di 12 mesi di stagionatura $(7,08$ e 2,78; rispettivamente). I campioni di crosta provenienti da forme che hanno subito il lavaggio ad acqua presenta un contenuto numericamente più alto di 3-cheto-4-colestene rispetto ai campioni di crosta provenienti da forme pulite mediante raschiatura a secco (7,73 e 2,88; rispettivamente).

\section{DISCUSSIONE}

Il primo dato interessante che emerge dai risultati presentati è che i campioni di crosta, rispetto ai campioni di pasta interna, evidenziano un contenuto di 3-cheto-4-colestene non trascurabile. Questo

\section{Tabella II: Rapporto area 3-cheto-4- colestene /area colesterolo $(\mathrm{m} / \mathrm{z}$ 124) per i campioni di pasta interna di Parmigiano Reggiano}

Table II: Area ratio 3-keto-4-cholestene/ cholesterol (m/z 124), Parmigiano Reggiano cheese samples

\begin{tabular}{cc} 
Campione & $\begin{array}{c}\text { area 3-cheto-4-colestene / } \\
\text { area colesterolo ( } \mathrm{m} / \mathrm{z} 124)\end{array}$ \\
\hline 32 & assente \\
\hline 33 & assente \\
\hline 34 & assente \\
\hline 35 & assente \\
\hline 36 & 0,04 \\
\hline 37 & assente \\
\hline 38 & assente \\
\hline 40 & assente \\
\hline 39 & assente \\
\hline
\end{tabular}

conferma quanto riportato nel precedente studio (2) e cioè che la pasta interna, protetta dall'ossidazione e dall'azione di enzimi di origine batterica e fungina che si sviluppano in superficie, non contiene 3-cheto-4-colestene.

Dai dati ottenuti, inoltre, è possibile ipotizzare una correlazione tra contenuto di 3-cheto-4-colestene e stagionatura del formaggio. Questo effetto è chiaramente sempre in relazione con la maggiore esposizione all'ossigeno e ad attività enzimatiche superficiali delle forme a stagionatura prolungata.

Grazie al campionamento effettuato è inoltre possibile ricavare un'indicazione preliminare sull'effetto della tipologia di pulizia della crosta del formaggio prima della grattugiatura. La raschiatura a secco determina una riduzione del contenuto di 3-cheto-4-colestene, probabilmente riconducibile alla rimozione superficiale di crosta che avviene nella pulizia mediante raschiatura, rispetto alla pulizia mediante lavaggio ad acqua che non porta alla rimozione dello strato superficiale di crosta. 


\section{Tabella III: Media e deviazione standard del rapporto area 3-cheto-4-colestene /area colesterolo (m/z 124) per i campioni di crosta analizzati di diverse stagionature (12-24 mesi) e sottoposti a diversi trattamenti di pulizia (a secco, ad acqua)}

Table III: Average and standard deviation area ratio 3-keto-4-cholestene/ cholesterol (m/z 124), rind samples from different age (12-24 months) and different cleaning (dry-cleaning, water cleaning)

\begin{tabular}{|c|c|c|c|}
\hline \multirow[t]{2}{*}{ Stagionatura } & \multirow[t]{2}{*}{ Pulizia forme } & \multicolumn{2}{|c|}{$\begin{array}{c}\text { area 3-cheto-4-colestene / area } \\
\text { colesterolo }(\mathrm{m} / \mathrm{z} 124)\end{array}$} \\
\hline & & Media & Dev. Standard \\
\hline 12 mesi & Raschiatura + Lavaggio $(\mathrm{n}=19)$ & 2,78 & 2,80 \\
\hline 24 mesi & Raschiatura + Lavaggio $(n=12)$ & 7,08 & 9,72 \\
\hline $12+24$ mesi $(n=21)$ & Raschiatura a secco & 2,88 & 2,48 \\
\hline $12+24$ mesi $(n=10)$ & Lavaggio ad acqua & 7,73 & 10,73 \\
\hline
\end{tabular}

I dati ottenuti risultano quindi interessanti nell'ottica di individuare un marker molecolare riconducibile alla presenza di crosta nei grattugiati. Tuttavia, a causa del ridotto numero di campioni analizzati in questo studio preliminare e dell'elevata variabilità dei dati per campioni all'interno di gruppi omogenei, non è stato possibile ottenere differenze statisticamente significative, ma è stato possibile ottenere indicazioni qualitative al momento preliminari. Sarà necessario incrementare il numero di campioni di diverse tipologie al fine di individuare i valori estremi dei range di variabilità del rapporto 3-cheto-4-colestene/colesterolo nei campioni di crosta, al fine poi di poter estendere il metodo a miscele di crosta e pasta con percentuali note.

\section{CONCLUSIONI}

Lo studio preliminare sulla presenza di 3-cheto-4-colestene in campioni di Parmigiano Reggiano ha permesso avere indicazioni sulle possibili variabili che portano a differenze nel contenuto di 3-cheto-4-colestene in Parmigiano Reggiano. È stato inoltre possibile ipotizzare una modalità analitica per distinguere tra Parmigiano Reggiano grattugiato prodotto utilizzando solo la pasta rispetto a Parmigiano Reggiano grattugiato prodotto a partire da una percentuale variabile di pasta interna e crosta.

\section{CONFLITTO DI INTERESSE}

Valentina Pizzamiglio e Marco Nocetti sono dipendenti del Consorzio del formaggio Parmigiano Reggiano che ha finanziato lo studio.

FINANZIAMENTO

Il presente studio è stato finanziato dal Consorzio del formaggio Parmigiano Reggiano.

\section{BIBLIOGRAFIA}

1. Disciplinare di produzione del formaggio Parmigiano Reggiano. www.parmigianoreggiano.it Ultimo accesso 15 settembre 2019.

2. Caligiani A, Lolli V, Marseglia A, Palla G. GC-MS quantificatification of ketocholestene in seasoned hard and semi-hard cheeses. $14^{\text {th }}$ Eurofed Lipids, Ghent, Book of Abstract.
3. Flanagan VP, Ferretti A; Schwartz DP, Ruth JM. Characterization of two steroidal ketones in dairy products. J Lipid Res 1975; 16(2):97-101.

4. Chiang YR, Ismail W, Gallien S, Heintz D, Van Dorsselaer A, Fuchs G. Cholest4-en-3-one-delta 1-dehydrogenase, a flavoprotein catalyzing the second step in anoxic cholesterol metabolism. Appl Environ Microbiol 2008; 74(1):107-13. 